\title{
ON THE WEAK SOLUTIONS OF AN OVERDETERMINED SYSTEM OF NONLINEAR FRACTIONAL PARTIAL INTEGRO-DIFFERENTIAL EQUATIONS
}

\author{
FATEMEH ABDOLRAZAGHI AND ABDOLRAHMAN RAZANI
}

Received 25 November, 2018

\begin{abstract}
In this research work, we employ the critical point theory based on the variational structure to prove the existence of at least three distinct weak solutions for an overdetermined system of nonlinear fractional partial Fredholm-Volterra integro-differential equations.
\end{abstract}

2010 Mathematics Subject Classification: 35J20; 34B15

Keywords: overdetermined system of differential equations, integral equation, Dirichlet condition, weak solution, critical point theory, variational method

\section{Preliminaries}

The study of partial differential equations (PDE's) started in the 18th century and it's area has been growing steadily in the past century. The analysis of physical models has remained to the present day one of the fundamental concerns of the development of PDE's. PDEs can be used for modelling a wide range of physical phenomena, encountered in statistical mechanics, mathematical physics, theoretical neuroscience, fluid dynamics and mathematical finance. On one side PDEs can be used to describe a wide variety of phenomena such as sound, heat, diffusion, electrostatics, electrodynamics, fluid dynamics, elasticity, or quantum mechanics. On the other side PDE's also became an essential tool in other branches of mathematics. Thus the theory of ordinary and partial differentia equations is a powerful theory to give a solution to engineering problems (see [22,23,25-28]). This theory can be generalized as theory of the overdetermined systems of differential and partial differential equations where it has been developed through some research works, e.g. [6, 10, 24, 30, 31,34]. On the other hand, to deal with the process having delay, it is not more convenient tool to apply an ordinary or partial differential equation. Instead, a convenient approach to resolve these kind of problems is to employ integro-differential equations. Many

This work is done when the second author is visiting University of Manitoba on a sabbatical leave from Imam Khomeini International University. 
mathematical models of physical phenomena lead to integro-differential equations $[2,3,8,9,15,29]$.

Consider the overdetermined system of nonlinear fractional partial Fredholm-Volterra integro-differential equations as follows:

$$
\left\{\begin{aligned}
{ }_{t} D_{T}^{\alpha_{i}} & \left(a_{i}(t)_{0} D_{t}^{\alpha_{i}} u_{i}(t)\right)=\lambda F_{u_{i}}\left(t, u_{1}(t), \cdots, u_{n}(t)\right) \\
& +\int_{0}^{T} k_{1, i}(t, \tau) h_{1, i}\left(u_{i}(\tau)\right) \mathrm{d} \tau \\
& +\int_{0}^{t} k_{2, i}(t, \tau) h_{2, i}\left(u_{i}(\tau)\right) \mathrm{d} \tau, \quad i=1, \cdots, n \\
u_{i}(t)= & f_{i}(t) u_{i}(t)+\int_{0}^{T} k_{1, i}(t, \tau) h_{1, i}\left(u_{i}(\tau)\right) \mathrm{d} \tau \\
& +\int_{0}^{t} k_{2, i}(t, \tau) h_{2, i}\left(u_{i}(\tau)\right) \mathrm{d} \tau, \quad i=1, \cdots, n \\
u_{i}(0)= & u_{i}(T)=0, \quad i=1, \cdots, n
\end{aligned}\right.
$$

where $t \in(0, T), \lambda$ is a positive real parameter, $0<\alpha_{i} \leq 1, a_{i}(t) \in L^{\infty}[0, T]$, $\bar{a}_{i}=\operatorname{essin}_{[0, T]} a_{i}(t)>0, f_{i}:[0, T] \rightarrow \mathbb{R}$ is a continuous function with the maximum norm $\left\|f_{i}\right\|_{\infty}=\max _{0 \leq t \leq T}\left|f_{i}(t)\right|=M_{i}^{f}, F:[0, T] \times \mathbb{R}^{n} \rightarrow \mathbb{R}$ is a function to the extent that $F\left(t, u_{1}, \cdots, u_{n}\right)$ is continuous with respect to $t$ and continuously differentiable with respect to $u_{i}$ i.e. $F\left(\cdot, u_{1}, \cdots, u_{n}\right) \in C[0, T]$ and $F(t, \cdot, \cdots, \cdot) \in C^{1}\left(\mathbb{R}^{n}\right)$, and further, $k_{1, i}(\cdot, \cdot), k_{2, i}(\cdot, \cdot) \in C([0, T],[0, T])$ hence the kernels $k_{1, i}$ and $k_{2, i}$ are bounded by for example $L_{i}$ and $M_{i}$, respectively. Also, we suppose $h_{1, i}, h_{2, i}: \mathbb{R} \rightarrow \mathbb{R}$ are Lipschitz continuous functions with the Lipschitz constants $L_{i}^{\prime}>0$ and $M_{i}^{\prime}>0$, i.e., $\left|h_{1, i}\left(\zeta_{1}\right)-h_{1, i}\left(\zeta_{2}\right)\right| \leq L_{i}^{\prime}\left|\zeta_{1}-\zeta_{2}\right|$ and $\left|h_{2, i}\left(\zeta_{1}\right)-h_{2, i}\left(\zeta_{2}\right)\right| \leq M_{i}^{\prime}\left|\zeta_{1}-\zeta_{2}\right|$ for every $\zeta_{1}, \zeta_{2} \in \mathbb{R}$ and $i=1, \cdots, n$. Moreover, $F_{s}$ denotes the partial derivative of $F$ with respect to $s$ and, ${ }_{t} D_{T}^{\gamma}$ and ${ }_{0} D_{t}^{\gamma}$ are the right and left Riemann-Liouville type of fractional derivatives of order $\gamma$ which are defined by [14]

$$
\begin{aligned}
& { }_{0} D_{t}^{\gamma} u(t)=\frac{1}{\Gamma(1-\gamma)} \frac{\mathrm{d}}{\mathrm{d} t} \int_{0}^{t} \frac{u(s)}{(t-s)^{\gamma}} \mathrm{d} s, \\
& { }_{t} D_{T}^{\gamma} u(t)=-\frac{1}{\Gamma(1-\gamma)} \frac{\mathrm{d}}{\mathrm{d} t} \int_{t}^{T} \frac{u(s)}{(s-t)^{\gamma}} \mathrm{d} s .
\end{aligned}
$$

The standard and typical approach to study the existence of solutions to the nonlinear fractional boundary value problems is the fixed point theory, see for example $[1,4,16,35]$. But, another well-developed, successful and recently more attracting method is the calculus of variation to investigate the existence of solutions to the differential equation with type of integer order and very recently fractional order, the readers are referred to $[7,11-13,17-21,32,33,36]$ and the references therein to attain more information about this approach.

Here, the well-known three critical points theorem due to Bonanno and Marano [5] is considered. Then the existence of at least three different weak solutions to the overdetermined system (1.1) is proved. In fact, the main steps to apply this theorem is to define two convenient functionals which satisfy all conditions of theorem's assumptions. 


\section{THREE DISTINCT WEAK SOLUTIONS}

In order to prove the existence of at least three weak solutions (1.1), some Theorems and Definitions are recalled in this literature.

Definition 1. Consider $0<\alpha_{i} \leq 1, i=1, \cdots, n$, the fractional derivative space $E_{0}^{\alpha_{i}}$ is defined by the closure of $C_{0}^{\infty}([0, T], \mathbb{R})$ i.e. $E_{0}^{\alpha_{i}}=\overline{C_{0}^{\infty}([0, T], \mathbb{R})}$ with respect to the following weighted norm

$$
\|u\|_{\alpha_{i}}=\left(\int_{0}^{T} a_{i}(t)\left|{ }_{0} D_{t}^{\alpha_{i}} u(t)\right|^{2} \mathrm{~d} t+\int_{0}^{T}|u(t)|^{2} \mathrm{~d} t\right)^{\frac{1}{2}},
$$

for all $u \in E_{0}^{\alpha_{i}}, i=1, \cdots, n$.

Lemma 1 ([11]). If $\frac{1}{2}<\alpha_{i} \leq 1$ then for all $u \in E_{0}^{\alpha_{i}}, i=1, \cdots, n$,

$$
\|u\|_{L^{2}} \leq \frac{T^{\alpha_{i}}}{\Gamma\left(\alpha_{i}+1\right)}\left\|_{0} D_{t}^{\alpha_{i}} u\right\|_{L^{2}}
$$

$$
\|u\|_{\infty} \leq \frac{T^{\alpha_{i}-\frac{1}{2}}}{\Gamma\left(\alpha_{i}\right) \sqrt{2 \alpha_{i}-1}}\left\|_{0} D_{t}^{\alpha_{i}} u\right\|_{L^{2}} .
$$

Remark 1. The following inequalities for $i=1, \cdots, n$, are straightforward from Lemma 1,

(1)

$$
\|u\|_{L^{2}} \leq \frac{T^{\alpha_{i}}}{\Gamma\left(\alpha_{i}+1\right) \sqrt{\bar{a}_{i}}}\left(\int_{0}^{T} a_{i}(t)\left|{ }_{0} D_{t}^{\alpha_{i}} u(t)\right|^{2} \mathrm{~d} t\right)^{\frac{1}{2}}
$$

$$
\|u\|_{\infty} \leq \frac{T^{\alpha_{i}-\frac{1}{2}}}{\Gamma\left(\alpha_{i}\right) \sqrt{\bar{a}_{i}\left(2 \alpha_{i}-1\right)}}\left(\int_{0}^{T} a_{i}(t)\left|{ }_{0} D_{t}^{\alpha_{i}} u(t)\right|^{2} \mathrm{~d} t\right)^{\frac{1}{2}} .
$$

Remark 2. The norm which is defined by equation (2.1) is equivalent to the following norm

$$
\|u\|_{\alpha_{i}}=\left(\left.\left.\int_{0}^{T} a_{i}(t)\right|_{0} D_{t}^{\alpha_{i}} u(t)\right|^{2} \mathrm{~d} t\right)^{\frac{1}{2}}, \text { for all } u \in E_{0}^{\alpha_{i}}, i=1, \cdots, n .
$$

The norm (2.6) is the norm that we need. Now, define $X=\prod_{i=1}^{i=n} E_{0}^{\alpha_{i}}$ augmented to the norm

$$
\|U\|_{X}=\sum_{i=1}^{n}\left\|u_{i}\right\|_{\alpha_{i}}, u_{i} \in E_{0}^{\alpha_{i}}, \quad U=\left(u_{1}, u_{2}, \cdots, u_{n}\right) \in X .
$$


Definition 2. $U=\left(u_{1}, u_{2}, \cdots, u_{n}\right) \in X$ is called the weak solution of the system (1.1) if the following equation satisfies

$$
\begin{aligned}
0= & \sum_{i=1}^{n} \int_{0}^{T} a_{i}(t)_{0} D_{t}^{\alpha_{i}} u_{i}(t)_{0} D_{t}^{\alpha_{i}} v_{i}(t) \mathrm{d} t \\
& -\sum_{i=1}^{n} \int_{0}^{T} \int_{0}^{T} k_{1, i}(t, \tau) h_{1, i}\left(u_{i}(\tau)\right) v_{i}(t) \mathrm{d} \tau \mathrm{d} t \\
& -\sum_{i=1}^{n} \int_{0}^{T} \int_{0}^{t} k_{2, i}(t, \tau) h_{2, i}\left(u_{i}(\tau)\right) v_{i}(t) \mathrm{d} \tau \mathrm{d} t \\
& -\lambda \int_{0}^{T} \sum_{i=1}^{n} F_{u_{i}}\left(t, u_{1}(t), \cdots, u_{n}(t)\right) v_{i}(t) \mathrm{d} t
\end{aligned}
$$

for every $V=\left(v_{1}, v_{2}, \cdots, v_{n}\right) \in X$.

Remark 3. Notice that we have used the number of $n$ equations of the system (1.1) to define the weak solution. In fact, we use the remain equations, the number of $n$ integral equations, directly to define an operator, in what follows, so that its Gâteaux derivative is some parts of the equation (2.8).

Definition 3. Define the operator $H_{i}: E_{0}^{\alpha_{i}} \rightarrow E_{0}^{\alpha_{i}}$ as

$$
\begin{aligned}
H_{i}\left(u_{i}(t)\right)= & \frac{1}{2} \int_{0}^{T} k_{1, i}(t, \tau) h_{1, i}\left(u_{i}(\tau)\right) u_{i}(t) \mathrm{d} \tau \\
& +\frac{1}{2} \int_{0}^{t} k_{2, i}(t, \tau) h_{2, i}\left(u_{i}(\tau)\right) u_{i}(t) \mathrm{d} \tau-\frac{1}{2} f_{i}(t)\left(u_{i}(t)\right)^{2},
\end{aligned}
$$

for all $t \in[0, T]$ and for $1 \leq i \leq n$.

From the system (1.1), it is straightforward to see

$$
\begin{aligned}
\left(u_{i}(t)+\theta v_{i}(t)\right)^{2}= & f_{i}(t)\left(u_{i}(t)+\theta v_{i}(t)\right)^{2} \\
& +\int_{0}^{T} k_{1, i}(t, \tau) h_{1, i}\left(u_{i}(\tau)+\theta v_{i}(\tau)\right)\left(u_{i}(t)+\theta v_{i}(t)\right) \mathrm{d} \tau \\
& +\int_{0}^{t} k_{2, i}(t, \tau) h_{2, i}\left(u_{i}(\tau)+\theta v_{i}(\tau)\right)\left(u_{i}(t)+\theta v_{i}(t)\right) \mathrm{d} \tau .
\end{aligned}
$$


The Gâteaux derivative of $H_{i}$ is

$$
\begin{aligned}
H_{i}^{\prime}\left(u_{i}(t)\right)\left(v_{i}(t)\right)= & \frac{\mathrm{d}}{\mathrm{d} \theta}\left\{\frac{1}{2} \int_{0}^{T} k_{1, i}(t, \tau) h_{1, i}\left(u_{i}(\tau)+\theta v_{i}(\tau)\right)\left(u_{i}(t)+\theta v_{i}(t)\right) \mathrm{d} \tau\right. \\
& +\frac{1}{2} \int_{0}^{t} k_{2, i}(t, \tau) h_{2, i}\left(u_{i}(\tau)+\theta v_{i}(\tau)\right)\left(u_{i}(t)+\theta v_{i}(t)\right) \mathrm{d} \tau \\
& \left.-\frac{1}{2} f_{i}(t)\left(u_{i}(t)+\theta v_{i}(t)\right)^{2}\right\}_{\theta=0} \\
= & \frac{\mathrm{d}}{\mathrm{d} \theta}\left\{\frac{1}{2}\left(u_{i}(t)+\theta v_{i}(t)\right)^{2}-\frac{1}{2} f_{i}(t)\left(u_{i}(t)+\theta v_{i}(t)\right)^{2}\right. \\
& \left.-\frac{1}{2} f_{i}(t)\left(u_{i}(t)+\theta v_{i}(t)\right)^{2}\right\} \\
= & u_{i}(t) v_{i}(t)-f_{i}(t) u_{i}(t) v_{i}(t) \\
= & \left(u_{i}(t)-f_{i}(t) u_{i}(t)\right) v_{i}(t) \\
= & \int_{0}^{T} k_{1, i}(t, \tau) h_{1, i}\left(u_{i}(\tau)\right) v_{i}(t) \mathrm{d} \tau \\
& +\int_{0}^{t} k_{2, i}(t, \tau) h_{2, i}\left(u_{i}(\tau)\right) v_{i}(t) \mathrm{d} \tau .
\end{aligned}
$$

The following theorem is recalled form [5] to obtain at least three distinct critical points.

Theorem 1 ([5, Theorem 3.6]). Let $X$ be a reflexive real Banach space and $\Phi: X \rightarrow \mathbb{R}$ a sequentially weakly lower semicontinuous, coercive and continuously Gâteaux differentiable in which its Gâteaux derivative has a continuous inverse on $X^{*}$, moreover, suppose that $\Psi: X \rightarrow \mathbb{R}$ be a sequentially weakly upper semicontinuous and continuously Gâteaux differentiable functional in which its Gâteaux derivative is compact, such that $\Phi(0)=\Psi(0)=0$. Suppose also there exist $r \in \mathbb{R}$ and $u_{1} \in X$ with $0<r<\Phi\left(u_{1}\right)$, satisfying

(1) $\sup _{\left.\left.u \in \Phi^{-1}(]-\infty, r\right]\right)} \Psi(u)<r \frac{\Psi\left(u_{1}\right)}{\Phi\left(u_{1}\right)}$

(2) for all $\left.\left.\lambda \in \Lambda_{r}:=\right] \frac{\Phi\left(u_{1}\right)}{\Psi\left(u_{1}\right)}, \frac{r}{\sup _{\left.\left.u \in \Phi^{-1}(]-\infty, r\right]\right)} \Psi(u)}\right]$, the functional $\Phi-\lambda \Psi$ is coercive.

Then for any $\lambda \in \Lambda_{r}$ the functional $\Phi-\lambda \Psi$ admits at least three distinct critical points in $X$.

The main result of this paper states the existence of at least different three weak solutions of the system (1.1). For convenience we set the following notations: $\theta_{i}=$ 


$$
\begin{aligned}
& \max \left\{L_{i} L_{i}^{\prime}, M_{i} M_{i}^{\prime}\right\}, \\
& \sigma=\min _{1 \leq i \leq n}\left\{\sigma_{i}\right\}, \sigma_{i}=1-\frac{2 \theta_{i} T^{2 \alpha_{i}+1}+M_{i}^{f} T^{2 \alpha_{i}}}{\left(\Gamma\left(\alpha_{i}\right)\right)^{2} \bar{a}_{i}\left(2 \alpha_{i}-1\right)}, \\
& M=\max _{1 \leq i \leq n}\left\{\frac{T^{2 \alpha_{i}-1}}{\left(\Gamma\left(\alpha_{i}\right)\right)^{2} \bar{a}_{i}\left(2 \alpha_{i}-1\right)-2 \theta_{i} T^{2 \alpha_{i}+1}-M_{i}^{f} T^{2 \alpha_{i}}}\right\}, \\
& \Upsilon(c)=\left\{\Xi=\left(\xi_{1}, \xi_{2}, \cdots, \xi_{n}\right) \in \mathbb{R}^{n}: \frac{1}{2} \sum_{i=1}^{n} \xi_{i}^{2} \leq c\right\}, c>0,
\end{aligned}
$$

and

$$
L=\max _{1 \leq i \leq n}\left\{\frac{T^{2 \alpha_{i}}}{\sigma\left(\Gamma\left(\alpha_{i}+1\right)\right)^{2} \bar{a}_{i}}\right\} .
$$

The main result is as follows:

Theorem 2. Suppose $F:[0, T] \times \mathbb{R}^{n} \rightarrow \mathbb{R}$ is a function such that $F\left(\cdot, u_{1}, \cdots, u_{n}\right) \in C[0, T], F(t, \cdot, \cdots, \cdot) \in C^{1}\left(\mathbb{R}^{n}\right)$ and $F(t, 0, \cdots, 0)=0$ for all $t \in$ $[0, T]$. Set

$$
\varrho:=\frac{\sum_{i=1}^{n}\left(\left\|\omega_{i}\right\|_{\alpha_{i}}^{2}-2 \int_{0}^{T} H_{i}\left(\omega_{i}(t)\right) d t\right)}{2 \int_{0}^{T} F\left(t, \omega_{1}(t), \cdots, \omega_{n}(t)\right) d t}
$$

and

$$
\vartheta:=\frac{r}{\int_{0}^{T} \sup _{\left(\xi_{1}, \cdots, \xi_{n}\right) \in \Upsilon(M r)} F\left(t, \xi_{1}, \cdots, \xi_{n}\right) d t} .
$$

If there exist a constant $r>0$ and a vector-valued function $\Omega(t)=\left(\omega_{1}(t), \cdots, \omega_{n}(t)\right)$ such that

(H0) $\frac{1}{2}<\alpha_{i} \leq 1$;

(H1) $\frac{2 \theta_{i} T^{2 \alpha_{i}+1}+M_{i}^{f} T^{2 \alpha_{i}}}{\left(\Gamma\left(\alpha_{i}\right)\right)^{2} \bar{a}_{i}\left(2 \alpha_{i}-1\right)}<1$;

(H2) $\sum_{i=1}^{n}\left\|\omega_{i}\right\|_{\alpha_{i}}^{2} \geq 2 r+2 \sum_{i=1}^{n} \int_{0}^{T} H_{i}\left(\omega_{i}(t)\right) d t$;

(H3) $\varrho<\vartheta$;

(H4) $\liminf _{\forall i:\left|\xi_{i}\right| \rightarrow+\infty} \frac{F\left(t, \xi_{1}, \cdots, \xi_{n}\right)}{\sum_{i=1}^{n}\left|\xi_{i}\right|^{2}}<\frac{1}{2 L \vartheta}$.

Then for any $\lambda \in \Lambda=] \varrho, \vartheta[$, the system (1.1) has at least three different weak solutions in $X$.

Proof. Consider the space $X=\prod_{i=1}^{i=n} E_{0}^{\alpha_{i}}$ equipped with the norm $\|U\|_{X}$ defined by (2.7) which is a reflexive and separable Banach space. For any given $U=\left(u_{1}(t), \cdots, u_{n}(t)\right) \in X$, we present the functionals $\Phi, \Psi: X \rightarrow \mathbb{R}$ as follows:

$$
\Phi(U)=\frac{1}{2} \sum_{i=1}^{n}\left\|u_{i}\right\|_{\alpha_{i}}^{2}-\sum_{i=1}^{n} \int_{0}^{T} H_{i}\left(u_{i}(t)\right) \mathrm{d} t,
$$


and

$$
\Psi(U)=\int_{0}^{T} F\left(t, u_{1}(t), \cdots, u_{n}(t)\right) \mathrm{d} t .
$$

These functionals are well-defined Gâteaux differentiable and for all $V=\left(v_{1}, v_{2}, \cdots, v_{n}\right) \in X$,

$$
\begin{aligned}
\Phi^{\prime}(U)(V)= & \sum_{i=1}^{n} \int_{0}^{T} a_{i}(t)_{0} D_{t}^{\alpha_{i}} u_{i}(t)_{0} D_{t}^{\alpha_{i}} v_{i}(t) \mathrm{d} t \\
& -\sum_{i=1}^{n} \int_{0}^{T} \int_{0}^{T} k_{1, i}(t, \tau) h_{1, i}\left(u_{i}(\tau)\right) v_{i}(t) \mathrm{d} \tau \mathrm{d} t \\
& -\sum_{i=1}^{n} \int_{0}^{T} \int_{0}^{t} k_{2, i}(t, \tau) h_{2, i}\left(u_{i}(\tau)\right) v_{i}(t) \mathrm{d} \tau \mathrm{d} t,
\end{aligned}
$$

and

$$
\Psi^{\prime}(U)(V)=\int_{0}^{T} \sum_{i=1}^{n} F_{u_{i}}\left(t, u_{1}(t), \cdots, u_{n}(t)\right) v_{i}(t) \mathrm{d} t .
$$

Notice that $\Phi^{\prime}(U), \Psi^{\prime}(U) \in X^{*}$ in where $X^{*}$ is dual space of $X$. It can be easily proved that the functional $\Phi$ is sequentially weakly lower semicontinuous and its Gâteaux derivative has a continuous inverse on $X^{*}$. Furthermore, since $\left|k_{1, i}(t, \tau)\right| \leq$ $L_{i}$ and $\left|k_{2, i}(t, \tau)\right| \leq M_{i}$ for $0 \leq t, \tau \leq T$ and besides, $h_{1, i}$ and $h_{2, i}$ are Lipschitz continuous functions with the Lipschitz constants $L_{i}^{\prime}>0$ and $M_{i}^{\prime}>0$, hence

$$
\begin{aligned}
H_{i}\left(u_{i}(t)\right)= & \frac{1}{2} \int_{0}^{T} k_{1, i}(t, \tau) h_{1, i}\left(u_{i}(\tau)\right) u_{i}(t) \mathrm{d} \tau \\
& +\frac{1}{2} \int_{0}^{t} k_{2, i}(t, \tau) h_{2, i}\left(u_{i}(\tau)\right) u_{i}(t) \mathrm{d} \tau-\frac{1}{2} f_{i}(t)\left(u_{i}(t)\right)^{2} \\
\leq & \frac{1}{2}\left|u_{i}(t)\right| T L_{i} L_{i}^{\prime}\left\|u_{i}\right\|_{\infty}+\frac{1}{2}\left|u_{i}(t)\right| t M_{i} M_{i}^{\prime}\left\|u_{i}\right\|_{\infty}+\frac{1}{2} M_{i}^{f}\left\|u_{i}\right\|_{\infty}^{2} \\
\leq & \frac{1}{2} T L_{i} L_{i}^{\prime}\left\|u_{i}\right\|_{\infty}^{2}+\frac{1}{2} T M_{i} M_{i}^{\prime}\left\|u_{i}\right\|_{\infty}^{2}+\frac{1}{2} M_{i}^{f}\left\|u_{i}\right\|_{\infty}^{2} \\
\leq & \theta_{i} T\left\|u_{i}\right\|_{\infty}^{2}+\frac{1}{2} M_{i}^{f}\left\|u_{i}\right\|_{\infty}^{2} \\
= & \left(\theta_{i} T+\frac{1}{2} M_{i}^{f}\right)\left\|u_{i}\right\|_{\infty}^{2} .
\end{aligned}
$$

Equations (2.5), (2.6) and (2.15) imply

$$
\Phi(U)=\frac{1}{2} \sum_{i=1}^{n}\left\|u_{i}\right\|_{\alpha_{i}}^{2}-\sum_{i=1}^{n} \int_{0}^{T} H_{i}\left(u_{i}(t)\right) \mathrm{d} t
$$




$$
\begin{aligned}
& \geq \frac{1}{2} \sum_{i=1}^{n}\left\|u_{i}\right\|_{\alpha_{i}}^{2}-\sum_{i=1}^{n} \int_{0}^{T}\left(\theta_{i} T+\frac{1}{2} M_{i}^{f}\right)\left\|u_{i}\right\|_{\infty}^{2} \mathrm{~d} t \\
& =\frac{1}{2} \sum_{i=1}^{n}\left\|u_{i}\right\|_{\alpha_{i}}^{2}-\sum_{i=1}^{n}\left(\theta_{i} T^{2}+\frac{1}{2} M_{i}^{f} T\right)\left\|u_{i}\right\|_{\infty}^{2} \\
& \geq \frac{1}{2} \sum_{i=1}^{n}\left\|u_{i}\right\|_{\alpha_{i}}^{2}-\sum_{i=1}^{n}\left(\theta_{i} T^{2}+\frac{1}{2} M_{i}^{f} T\right) \frac{T^{2 \alpha_{i}-1}}{\left(\Gamma\left(\alpha_{i}\right)\right)^{2} \bar{a}_{i}\left(2 \alpha_{i}-1\right)}\left\|u_{i}\right\|_{\alpha_{i}}^{2} \\
& =\frac{1}{2} \sum_{i=1}^{n}\left(1-\frac{2 \theta_{i} T^{2 \alpha_{i}+1}+M_{i}^{f} T^{2 \alpha_{i}}}{\left(\Gamma\left(\alpha_{i}\right)\right)^{2} \bar{a}_{i}\left(2 \alpha_{i}-1\right)}\right)\left\|u_{i}\right\|_{\alpha_{i}}^{2} \\
& =\frac{1}{2} \sum_{i=1}^{n} \sigma_{i}\left\|u_{i}\right\|_{\alpha_{i}}^{2} \\
& \geq \frac{\sigma}{2} \sum_{i=1}^{n}\left\|u_{i}\right\|_{\alpha_{i}}^{2} \\
& =\frac{\sigma}{2}\|U\|_{X},
\end{aligned}
$$

we know that $\sigma$ is positive because each $\sigma_{i}$ is positive by assumption (H1), thus $\lim _{\|U\|_{X} \rightarrow+\infty} \Phi(U)=+\infty$ hence it is coercive. Let us to prove the functional $\Psi$ is definitely sequentially weakly upper semicontinuous and its derivative $\Psi^{\prime}: X \rightarrow X^{*}$ is a compact operator. If $\lim _{m \rightarrow+\infty} U_{m} \rightarrow U$ in $X$ where $U_{m}(t)=\left(u_{m, 1}(t), \cdots, u_{m, n}(t)\right)$, then absolutely $U_{m}$ converges uniformly to $U$ on the interval $[0, T]$, then

$$
\begin{aligned}
\limsup _{m \rightarrow+\infty} \Psi\left(U_{m}\right) & \leq \int_{0}^{T} \limsup _{m \rightarrow+\infty} F\left(t, u_{m, 1}(t), \cdots, u_{m, n}(t)\right) \mathrm{d} t \\
& =\int_{0}^{T} F\left(t, u_{1}(t), \cdots, u_{n}(t)\right) \mathrm{d} t \\
& =\Psi(U) .
\end{aligned}
$$

Hence $\Psi$ is sequentially weakly upper semicontinuous. Moreover, since $F(t, \cdot, \cdots, \cdot) \in C^{1}\left(\mathbb{R}^{n}\right)$ then for all $t \in[0, T]$

$$
\lim _{m \rightarrow+\infty} F\left(t, u_{m, 1}(t), \cdots, u_{m, n}(t)\right)=F\left(t, u_{1}(t), \cdots, u_{n}(t)\right)
$$

Now, the Lebesgue control convergence theorem yields $\Psi^{\prime}\left(U_{m}\right) \rightarrow \Psi^{\prime}(U)$ strongly, so it results in that $\Psi^{\prime}$ is strongly continuous on $X$. Therefore, $\Psi^{\prime}: X \rightarrow X^{*}$ is a compact operator. 
Suppose that $U_{0}(t)=(0, \cdots, 0)$ and $U_{1}(t)=\Omega(t)$ then

$$
\Phi\left(U_{0}(t)\right)=\Psi\left(U_{0}(t)\right)=0
$$

and the assumption (H2) yields directly

$$
0<r \leq \frac{1}{2} \sum_{i=1}^{n}\left\|\omega_{i}\right\|_{\alpha_{i}}^{2}-\sum_{i=1}^{n} \int_{0}^{T} H_{i}\left(\omega_{i}(t)\right) \mathrm{d} t=\Phi\left(U_{1}\right) .
$$

Equations (2.5), (2.6), (2.12) and (2.13), imply

$$
\begin{aligned}
& \left.\left.\Phi^{-1}(]-\infty, r\right]\right)=\{U \in X: \Phi(U) \leq r\} \\
& =\left\{U \in X: \frac{1}{2} \sum_{i=1}^{n}\left\|u_{i}\right\|_{\alpha_{i}}^{2}-\sum_{i=1}^{n} \int_{0}^{T} H_{i}\left(u_{i}(t)\right) \mathrm{d} t \leq r\right\} \\
& \subseteq\{U \in X: \\
& \left.\frac{1}{2} \sum_{i=1}^{n}\left\|u_{i}\right\|_{\alpha_{i}}^{2}-\sum_{i=1}^{n} \int_{0}^{T}\left(\theta_{i} T+\frac{1}{2} M_{i}^{f}\right)\left\|u_{i}\right\|_{\infty}^{2} \mathrm{~d} t \leq r\right\} \\
& =\left\{U \in X: \sum_{i=1}^{n} \frac{\left(\Gamma\left(\alpha_{i}\right)\right)^{2} \bar{a}_{i}\left(2 \alpha_{i}-1\right)}{2 T^{2 \alpha_{i}-1}}\left\|u_{i}\right\|_{\infty}^{2}\right. \\
& \left.-\sum_{i=1}^{n}\left(\theta_{i} T^{2}+\frac{1}{2} M_{i}^{f} T\right)\left\|u_{i}\right\|_{\infty}^{2} \leq r\right\} \\
& =\{U \in X: \\
& \left.\sum_{i=1}^{n} \frac{\left(\Gamma\left(\alpha_{i}\right)\right)^{2} \bar{a}_{i}\left(2 \alpha_{i}-1\right)-2 \theta_{i} T^{2 \alpha_{i}+1}-M_{i}^{f} T^{2 \alpha_{i}}}{2 T^{2 \alpha_{i}-1}}\left\|u_{i}\right\|_{\infty}^{2} \leq r\right\} \\
& \subseteq\left\{U \in X: \frac{1}{2 M} \sum_{i=1}^{n}\left\|u_{i}\right\|_{\infty}^{2} \leq r\right\} \\
& \subseteq\{U \in X: \\
& \left.\frac{1}{2} \sum_{i=1}^{n}\left|u_{i}(t)\right|^{2} \leq M r, \text { for all } t \in[0, T]\right\} \\
& \subseteq \Upsilon(M r)
\end{aligned}
$$


which leads to the following relation

$$
\begin{aligned}
\sup _{\left.\left.U \in \Phi^{-1}(]-\infty, r\right]\right)} \Psi(U) & =\sup _{\left.\left.U \in \Phi^{-1}(]-\infty, r\right]\right)} \int_{0}^{T} F\left(t, u_{1}(t), \cdots, u_{n}(t)\right) \mathrm{d} t \\
& \leq \sup _{\Xi \in \Upsilon(M r)} \int_{0}^{T} F\left(t, \xi_{1}, \cdots, \xi_{n}\right) \mathrm{d} t \\
& =\int_{0}^{T} \sup _{\Xi \in \Upsilon(M r)} F\left(t, \xi_{1}, \cdots, \xi_{n}\right) \mathrm{d} .
\end{aligned}
$$

By (H3), we have

$$
\begin{aligned}
\frac{\sup _{\left.\left.U \in \Phi^{-1}(]-\infty, r\right]\right)} \Psi(U)}{r} & =\frac{\sup _{\left.\left.U \in \Phi^{-1}(]-\infty, r\right]\right)} \int_{0}^{T} F\left(t, u_{1}(t), \cdots, u_{n}(t)\right) \mathrm{d} t}{r} \\
& \leq \frac{\int_{0}^{T} \sup _{\Xi \in \Upsilon(M r)} F\left(t, \xi_{1}, \cdots, \xi_{n}\right) \mathrm{d} t}{r} \\
& <\frac{2 \int_{0}^{T} F\left(t, \omega_{1}(t), \cdots, \omega_{n}(t)\right) \mathrm{d} t}{\sum_{i=1}^{n}\left\|\omega_{i}\right\|_{\alpha_{i}}^{2}-2 \sum_{i=1}^{n} \int_{0}^{T} H_{i}\left(\omega_{i}(t)\right) \mathrm{d} t} \\
& =\frac{\Psi(\Omega(t))}{\Phi(\Omega(t))} \\
& =\frac{\Psi\left(U_{1}\right)}{\Phi\left(U_{1}\right)},
\end{aligned}
$$

thus, $\sup _{\left.\left.U \in \Phi^{-1}(]-\infty, r\right]\right)} \Psi(U)<r \frac{\Psi\left(U_{1}\right)}{\Phi\left(U_{1}\right)}$ and hence the hypothesis (1) of Theorem 1 is valid.

By assumption (H4) there exits two constants $\mu, \varepsilon \in \mathbb{R}$ with the properties

$$
\begin{gathered}
\frac{\mu}{\sigma}<\frac{\int_{0}^{T} \sup _{\Xi \in \Upsilon(M r)} F\left(t, \xi_{1}, \cdots, \xi_{n}\right) \mathrm{d} t}{r}, \\
\text { and for all } \Xi \in \mathbb{R}^{n}: F\left(t, \xi_{1}, \cdots, \xi_{n}\right) \leq \frac{\mu}{2 L \sigma} \sum_{i=1}^{n}\left|\xi_{i}\right|^{2}+\varepsilon,
\end{gathered}
$$

for all $t \in[0, T]$. Then clearly for a fixed $U=\left(u_{1}(t), \cdots, u_{n}(t)\right) \in X$, we have

$$
F\left(t, u_{1}(t), \cdots, u_{n}(t)\right) \leq \frac{\mu}{2 L \sigma} \sum_{i=1}^{n}\left|u_{i}(t)\right|^{2}+\varepsilon,
$$

for all $t \in[0, T]$. Now, in the next step we have to show the coercivity of the functional $\Phi(U)-\lambda \Psi(U)$, suppose $\lambda \in \Lambda$, then bringing into accounts (2.4), (2.5), (2.14), (2.19) and (2.20), we have 


$$
\begin{aligned}
\Phi(U)-\lambda \Psi(U)= & \frac{1}{2} \sum_{i=1}^{n}\left\|u_{i}\right\|_{\alpha_{i}}^{2} \\
& -\sum_{i=1}^{n} \int_{0}^{T} H_{i}\left(u_{i}(t)\right) \mathrm{d} t-\lambda \int_{0}^{T} F\left(t, u_{1}(t), \cdots, u_{n}(t)\right) \mathrm{d} t \\
\geq & \frac{1}{2} \sum_{i=1}^{n}\left\|u_{i}\right\|_{\alpha_{i}}^{2}-\sum_{i=1}^{n} \int_{0}^{T}\left(\theta_{i} T+\frac{1}{2} M_{i}^{f}\right)\left\|u_{i}\right\|_{\infty}^{2} \mathrm{~d} t \\
& -\lambda \int_{0}^{T} F\left(t, u_{1}(t), \cdots, u_{n}(t)\right) \mathrm{d} t \\
\geq & \frac{1}{2} \sum_{i=1}^{n}\left\|u_{i}\right\|_{\alpha_{i}}^{2}-\sum_{i=1}^{n}\left(\theta_{i} T^{2}+\frac{1}{2} M_{i}^{f} T\right)\left\|u_{i}\right\|_{\infty}^{2} \mathrm{~d} t \\
& -\frac{\lambda \mu}{2 L \sigma} \int_{0}^{T}\left(\sum_{i=1}^{n}\left|u_{i}(t)\right|^{2}\right) \mathrm{d} t-\lambda T \varepsilon \\
\geq & \frac{1}{2} \sum_{i=1}^{n}\left\|u_{i}\right\|_{\alpha_{i}}^{2}-\sum_{i=1}^{n}\left(\theta_{i} T^{2}+\frac{1}{2} M_{i}^{f} T\right) \frac{T^{2 \alpha_{i}-1}}{\left(\Gamma\left(\alpha_{i}\right)\right)^{2} \bar{a}_{i}\left(2 \alpha_{i}-1\right)}\left\|u_{i}\right\|_{\alpha_{i}}^{2} \\
& -\frac{\lambda \mu}{2 L \sigma} \sum_{i=1}^{n} \frac{T^{2 \alpha_{i}}}{\left(\Gamma\left(\alpha_{i}+1\right)\right)^{2} \bar{a}_{i}}\left\|u_{i}\right\|_{\alpha_{i}}^{2}-\lambda T \varepsilon \\
= & \frac{1}{2} \sum_{i=1}^{n} \sigma_{i}\left\|_{i}\right\|_{\alpha_{i}}^{2}-\frac{\lambda \mu}{2 L \sigma} \sum_{i=1}^{n} \frac{T^{2 \alpha_{i}}}{\left(\Gamma\left(\alpha_{i}+1\right)\right)^{2} \bar{a}_{i}}\left\|u_{i}\right\|_{\alpha_{i}}^{2}-\lambda T \varepsilon \\
\geq & \frac{1}{2} \sum_{i=1}^{n} \sigma\left\|u_{i}\right\|_{\alpha_{i}}^{2}-\frac{\lambda \mu}{2} \sum_{i=1}^{n}\left\|u_{i}\right\|_{\alpha_{i}}^{2}-\lambda T \varepsilon \\
\geq & \frac{1}{2}\left(\sigma-\frac{\mu r}{\int_{0}^{T} \sup _{\Xi \in \Upsilon}(M r)} F\left(t, \xi_{1}, \cdots, \xi_{n}\right) \mathrm{d} t\right) \sum_{i=1}^{n}\left\|u_{i}\right\|_{\alpha_{i}}^{2}-\lambda T \varepsilon
\end{aligned}
$$

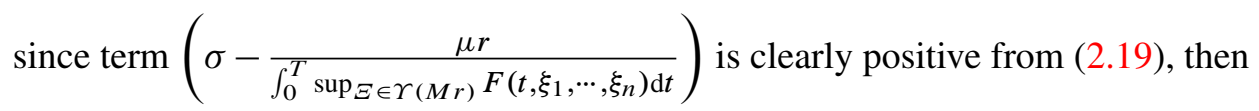

$$
\lim _{\|U\|_{X} \rightarrow+\infty}(\Phi(U)-\lambda \Psi(U))=+\infty .
$$

Therefore, it is verified that $\Phi-\lambda \Psi$ is coercive and then the hypothesis (2) of Theorem 1 is also established. Applying Theorem 1 and taking into account that the weak solutions of the system (1.1) are exactly constructed through the equation 
$\Phi^{\prime}(U)-\lambda \Psi^{\prime}(U)=0$, we conclude that the system (1.1) has at least three distinct weak solutions in $X$ for $\lambda \in \Lambda$ and then the proof is completed.

\section{ACKNOWLEDGMENTS}

The authors are grateful to anonymous reviewers for carefully reading this paper and for their valuable comments and suggestions, which have improved the paper.

\section{REFERENCES}

[1] R. P. Agarwal, M. Benchohra, and S. Hamani, "A survey on existence results for boundary value problems of nonlinear fractional differential equations and inclusions." Acta Appl. Math., vol. 109, no. 3, pp. 973-1033, 2010, doi: 10.1007/s10440-008-9356-6.

[2] B. Ahmad and J. J. Nieto, "Riemann-Liouville fractional integro-differential equations with fractional nonlocal integral boundary conditions.” Bound. Value Probl., vol. 2011, no. 1, p. 36, 2011, doi: 10.1186/1687-2770-2011-36.

[3] H. Aydi, A. Felhi, E. Karapinar, and S. Sahmim, "A Nadler-type fixed point theorem in dislocated spaces and applications." Miskolc Math. Notes, vol. 19, no. 1, pp. 111-124, 2018, doi: 10.18514/MMN.2018.1652.

[4] M. Benchohra, S. Hamani, and S. Ntouyas, "Boundary value problems for differential equations with fractional order and nonlocal conditions." Nonlinear Anal., vol. 71, no. 7, pp. 2391-2396, 2009, doi: 10.1016/j.na.2009.01.073.

[5] G. Bonanno and S. A. Marano, "On the structure of the critical set of non-differentiable functions with a weak compactness condition." Appl. Anal., vol. 89, no. 1, pp. 1-10, 2010, doi: 10.1080/00036810903397438.

[6] D. Ehsani, M. R. Mokhtarzadeh, and A. Razani, "Expansions of Dirichlet to Neumann operators under perturbations." Appl. Anal., vol. 89, no. 5, pp. 789-799, 2010, doi: 10.1080/00036811003649108.

[7] A. Firouzjai, G. A. Afrouzi, and S. Talebi, "Existence results for Kirchhoff type systems with singular nonlinearity." Opuscula Math., vol. 38, no. 2, pp. 187-199, 2018, doi: 10.7494/OpMath.2018.38.2.187.

[8] S. Gala, Q. Liu, and M. A. Ragusa, "Logarithmically improved regularity criterion for the nematic liquid crystal flows in $\dot{\mathrm{B}}_{\infty, \infty}^{-1}$ space." Comput. Math. Appl., vol. 65, no. 11, pp. 1738-1745, 2013, doi: 10.1016/j.camwa.2013.04.003.

[9] S. Gala, M. A. Ragusa, Y. Sawano, and H. Tanaka, "Uniqueness criterion of weak solutions for the dissipative quasi-geostrophic equations in Orlicz-Morrey spaces." Appl. Anal., vol. 93, no. 2, pp. 356-368, 2014, doi: 10.1080/00036811.2013.772582.

[10] Z. Goodarzi and A. Razani, "A periodic solution of the generalized forced Liénard equation." Abstr. Appl. Anal., vol. 2014, no. 132450, p. 5 pages, 2014, doi: 10.1155/2014/132450.

[11] F. Jiao and Y. Zhou, "Existence results for fractional boundary value problem via critical point theory." Internat. J. Bifur. Chaos Appl. Sci. Engrg., vol. 22, no. 04, 2012, doi: 10.1142/S0218127412500861.

[12] S. M. Khalkhali, S. Heidarkhani, and A. Razani, "Infinitely many solutions for a fourth-order boundary-value problem." Electron. J. Differential Equations, vol. 164, pp. 1-14, 2012.

[13] S. M. Khalkhali and A. Razani, "Multiple solutions for a quasilinear $(p, q)$-elliptic system." Electron. J. Differential Equations, vol. 2013, no. 144, pp. 1-14, 2013.

[14] A. A. Kilbas, H. M. Srivastava, and J. J. Trujillo, Theory and Applications of Fractional Differential Equations. $\quad$ Elsevier Science, Amsterdam, 2006. 
[15] N. Kouhestani and A. Moameni, "Multiplicity results for elliptic problems with super-critical concave and convex nonlinearties." Calc. Var. Partial Differential Equations, vol. 57, no. 2, p. 54, 2018, doi: 10.1007/s00526-018-1333-y.

[16] C. Li, R. P. Agarwal, and D.-L. Wu, "Existence and multiplicity of solutions for a class of superlinear elliptic systems.” Adv. Nonlinear Anal., vol. 7, no. 2, pp. 183-196, 2018, doi: 10.1515/anona2016-0045.

[17] Y.-N. Li, H.-R. Sun, and Q.-G. Zhang, "Existence of solutions to fractional boundary-value problems with a parameter." Electron. J. Differential Equations, vol. 2013, no. 141, pp. 1-12, 2013.

[18] R. Mahdavi Khanghahi and A. Razani, "Solutions for a singular elliptic problem involving the $p(x)$-Laplacian." Filomat, vol. 32, no. 14, 2018.

[19] M. Makvand Chaharlang and A. Razani, "Infinitely many solutions for a fourth order singular elliptic problem." vol. 32, no. 14, 2018.

[20] M. Makvand Chaharlang and A. Razani, "Existence of infinitely many solutions for a class of nonlocal problems with Dirichlet boundary condition." Commun. Korean Math. Soc., vol. 34, no. 1, pp. 155-167, 2019, doi: 10.4134/CKMS.c170456.

[21] M. Makvand Chaharlang and A. Razani, "A fourth order singular elliptic problem involving $p$ biharmonic operator.” Taiwanese J. Math., Accepted, doi: 10.11650/tjm/180906.

[22] M. R. Mokhtarzadeh, M. R. Pournaki, and A. Razani, "An existence-uniqueness theorem for a class of boundary value problems." vol. 13, no. 2, pp. 583-592, 2012.

[23] M. R. Pournaki and A. Razani, "On the existence of periodic solutions for a class of generalized forced Liénard equations." Appl. Math. Lett., vol. 20, pp. 248-254, 2007, doi: 10.1016/j.aml.2006.06.004.

[24] D. G. Quillen, Formal properties overdetermined system of linear partial differential equations. Harvard University, 1964.

[25] A. Razani, "Shock waves in gas dynamics." Surv. Math. Appl., vol. 2, pp. 59-89, 2007.

[26] A. Razani, "An existence theorem for ordinary differential equation in Menger probabilistic metric space.” Miskolc Math. Notes, vol. 15, no. 2, pp. 711-716, 2014, doi: 10.18514/MMN.2014.640.

[27] A. Razani, "Chapman-Jouguet travelling wave for a two-steps reaction scheme." Ital. J. Pure Appl. Math., vol. 39, pp. 544-553, 2018.

[28] A. Razani, "Subsonic detonation waves in porous media." Phys. Scr., 2019, doi: 10.1088/14024896/ab029b.

[29] A. Samadi, "Applications of meaure of noncomactness to coupled fixed points and systems of integral equations." Miskolc Math. Notes, vol. 19, no. 1, pp. 537-553, 2018, doi: 10.18514/MMN.2018.2532.

[30] E. Shivanian, "Analysis of meshless local radial point interpolation (MLRPI) on a nonlinear partial integro-differential equation arising in population dynamics.” Eng. Anal. Bound. Elem., vol. 37, no. 12, pp. 1693-1702, 2013, doi: 10.1016/j.enganabound.2013.10.002.

[31] D. C. Spencer, "Overdetermined systems of linear partial differential equations." Bull. Amer. Math. Soc. (N.S.), vol. 75, no. 2, pp. 179-239, 1969.

[32] H.-R. Sun and Q.-G. Zhang, "Existence of solutions for a fractional boundary value problem via the Mountain Pass method and an iterative technique." Comput. Math. Appl., vol. 64, no. 10, pp. 3436-3443, 2012, doi: 10.1016/j.camwa.2012.02.023.

[33] W. Xie, J. Xiao, and Z. Luo, "Existence of solutions for fractional boundary value problem with nonlinear derivative dependence." in Abstract and Applied Analysis, vol. 2014, doi: 10.1155/2014/812910, 2014.

[34] M. Zaytsev and V. Akkerman, "Reduction of over-determined systems of differential equations." 2012. 
[35] S. Zhang, "Positive solutions to singular boundary value problem for nonlinear fractional differential equation." Comput. Math. Appl., vol. 59, no. 3, pp. 1300-1309, 2010, doi: 10.1016/j.camwa.2009.06.034.

[36] Y. Zhao, H. Chen, and B. Qin, "Multiple solutions for a coupled system of nonlinear fractional differential equations via variational methods." Appl. Math. Comput., vol. 257, pp. 417-427, 2015, doi: 10.1016/j.amc.2014.12.128.

\section{Authors' addresses}

\section{Fatemeh Abdolrazaghi}

Imam Khomeini International University, Faculty of Science, Department of Pure Mathematics, Postal code: 34149-16818, Qazvin, Iran

E-mail address: fatemeh_abdolrazaghi@yahoo.com

\section{Abdolrahman Razani}

Imam Khomeini International University, Faculty of Science, Department of Pure Mathematics, Postal code: 34149-16818, Qazvin, Iran

E-mail address: razaniesci.ikiu.ac.ir 